\title{
Current achievements of the DELPHI Ring Imaging Cherenkov detector
}

\author{
W. Adam et al \\ Presented by M. Dracos
}

\begin{abstract}
The DELPHI experiment has already collected 2.5 million $Z^{0}$ decays with the Ring Imaging Cherenkov detector (RICH) operational. This detector, covering most of the solid angle, is designed to perform $\pi / K$ separation from 0.8 to $20 \mathrm{GeV} / \mathrm{c}$ and $\mathrm{K} / \mathrm{p}$ separation from 0.8 to $35 \mathrm{GeV} / \mathrm{c}$. After a brief detector description we discuss the actual operating conditions, the data monitoring and the signal treatment. The collected data from $Z^{0}$ decays and the detector response to signals from the calibration system are used to evalute the performance of the RICH system.
\end{abstract}





\title{
CURRENT ACHIEVEMENTS OF THE DELPHI RING IMAGING CHERENKOV DETECTOR
}

\author{
Presented by Marcos DRACOS
}

W. Adam $^{3}$, E. Albrecht ${ }^{3}$, D. Allen ${ }^{3}$, M-L. Andrieux ${ }^{7}$, G. van Apeldoorn ${ }^{12}$, C. Aubret ${ }^{2}$, A. Augustinus ${ }^{12}$, P. Baillon ${ }^{3}$ M. Battaglia ${ }^{15}$, D. Bloch ${ }^{4}$, O. Botner ${ }^{16}$, C. Bourdarios ${ }^{3}$, J. M. Brunet ${ }^{2}$, A. Buys ${ }^{3}$, P. Carrie ${ }^{3}$, P. Cavalli ${ }^{7}$, G. Cerutti ${ }^{3}$, M. Chevry ${ }^{10}$, E. Christophel ${ }^{4}$, E. Dahl-Jensen ${ }^{11}$, G. Damgaard ${ }^{11}$, N. Dimitriou ${ }^{5}$, B. D'Almagne ${ }^{8}$, M. Davenport ${ }^{3}$, F. Delbecque ${ }^{3}$, J. Dolbeau ${ }^{2}$, M. Dracos ${ }^{4}$, M. Dris ${ }^{13}$, T. Ekelöf ${ }^{16}$, J. P. Engel ${ }^{4}$, D. Fassouliotis ${ }^{13}$, T. A. Filippas ${ }^{13}$, E. Fokitis ${ }^{13}$, F. Fontanelli ${ }^{6}$, A. Fontenille ${ }^{7}$, D. Fraissard ${ }^{3}$, H. Fürstenau ${ }^{3}$, J. Garcia ${ }^{14}$, E. N. Gazis ${ }^{13}$, D. Gillespie ${ }^{3}$, V. Gracco ${ }^{6}$, L. Guglielmi ${ }^{2}$, F. Hahn ${ }^{3}$, S. Haider ${ }^{12}$, A. Hallgren ${ }^{16}$, W. Hao' ${ }^{12}$ K. Huet ${ }^{10}$, P. Ioannou ${ }^{1}$, S. Mie ${ }^{3}$, S. Jääskeläinen ${ }^{3}$, C. Joram ${ }^{3}$, P. Juillot ${ }^{4}$, E. Karvelas ${ }^{5}$, S. Katsanevas ${ }^{1}$, E. Katsoufis ${ }^{13}$, N. Kjaer ${ }^{11}$, P. M. Kluit ${ }^{12}$, B. Koene ${ }^{12}$, C. Kourkoumelis ${ }^{1}$, G. Lecoeur ${ }^{3}$, G. Lenzen ${ }^{17}$, L-E. Lindqvist ${ }^{16}$, A. López Agúera ${ }^{14}$, D. Loukas ${ }^{5}$, S. Maltezos ${ }^{13}$, A. Markou ${ }^{5}$, J. Medbo ${ }^{16}$, J. Michalowski ${ }^{9}$, F. Montano ${ }^{6}$, G. Mourgue ${ }^{3}$, B. S. Nielsen ${ }^{3,11}$, R. Nicolaidou ${ }^{1}$, Th. D. Papadopoulou ${ }^{13}$, A. Petrolini ${ }^{6}$, D. Poutot ${ }^{2}$, G. Polok ${ }^{9}$, H. Rahmani ${ }^{13}$, M. Reale ${ }^{17}$, L. K. Resvanis ${ }^{1}$, G. Sajot $^{7}$, M. Sannino ${ }^{6}$, E. Schyns ${ }^{17}$, S. Squarcia ${ }^{6}$, P. Stassi ${ }^{7}$, R. Strub ${ }^{4}$, J. Thadome ${ }^{17}$, G. E. Theodosiou ${ }^{5}{ }^{\dagger}$, M. J. Tobar ${ }^{3}$, D. Z. Toet ${ }^{12}$, L. Traspedini ${ }^{6}$, G. Tripodi', G. Tristram ${ }^{2}$, A. Tsirou ${ }^{3}$, O. Ullaland ${ }^{3}$ and E. Zevgolatakos ${ }^{*}$

The DELPHI experiment has already collected 2.5 million $\mathrm{Z}^{0}$ decays with the Ring Imaging Cherenkov detector (RICH) operational. This detector, covering most of the solid angle, is designed to perform $\pi / \mathrm{K}$ separation from 0.8 to $20 \mathrm{GeV} / c$ and $\mathrm{K} / \mathrm{p}$ separation from 0.8 to $35 \mathrm{GeV} / c$. After a brief detector description we discuss the actual operating conditions, the data monitoring and the signal treatment. The collected data from $\mathrm{Z}^{0}$ decays and the detector response to signals from the calibration system are used to evaluate the performance of the RICH system.

\begin{abstract}
*1 University of Athens, Physics Department, Physics Laboratory, Solonos Str. 104, GR-10680 Athens, Greece. ${ }^{2}$ Collège de France, Laboratoire de Physique Corpusculaire, 11 place Marcelin-Berthelot, F-75231 Paris, France. ${ }^{3}$ CERN, CH-1211 Geneva 23, Switzerland. ${ }^{4}$ Centre de Recherche Nucléaire, IN2P3-CNRS/ULP, BP28, F67037 Strasbourg Cédex2, France. ${ }^{5}$ Institute of Nuclear Physics, N.C.S.R. 'Demokritos', P.O. Box 60228, GR-15310 Aghia Paraskevi, Attiki, Greece. ${ }^{6}$ Dipartimento di Fisica, Università di Genova and INFN, Via Dodecaneso 33, I-16146 Genova, Italy. ${ }^{7}$ Institut des Sciences Nucléaires, Université de Grenoble 1, F-38026 Grenoble, France. ${ }^{8}$ LAL Laboratoire de l'Accélérateur Linéaire, Université de ParisSud (Paris XI), Bâtiment 200, F-91405 Orsay Cédex, France. ${ }^{9}$ High Energy Physics Laboratory, Inst. of Nucl. Physics, Ul. Kawiory 26a, PL-30055 Krakow 30, Poland. ${ }^{10}$ Université de Mons-Hainaut, Service de Physique des Particules Elémentaires, Faculté des Sciences, Av. Maistriau 15, B-7000 Mons, Belgium. ${ }^{11}$ Niels Bohr Institute, Blegdamsvej 17, DK-2100 Copenhagen, Denmark. ${ }^{12}$ NIKHEF-H, Postbus 41882, NL-1009 DB Amsterdam, The Netherlands. ${ }^{13}$ NTU National Technical University, Physics Laboratory II, 9 Heroes of Polytechneion Street, Zografou, GR-15780 Athens, Greece. ${ }^{14}$ Facultad de Ciencias, Universidad de Santander, av. de los Castros, E39005 Santander, Spain. ${ }^{15}$ Research Institute for High Energy Physics, SEFT, Siltavuorenpenger 20c, SF-00170 Helsinki, Finland. ${ }^{16}$ Dept. of Radiation Sciences, University of Uppsala, P.O. Box 535, S-751 21 Uppsala, Sweden. ${ }^{17}$ Fachbereich Physik, University of Wuppertal, GaussStr. 20, D-42097 Wuppertal, Germany. $\quad$ Deceased.
\end{abstract}

\section{INTRODUCTION}

The DELPHI Ring Imaging CHerenkov detector (RICH) has been designed to identify charged particles over a large momentum range $(0.8-$ $35 \mathrm{GeV} / \mathrm{c}$ ). A Barrel and a Forward RICH are used with fiducial coverage of $73 \%$ of the total solid angle. A detailed description of these two RICH detectors can be found in $[1,2]$.

For low momenta particles $(p<5 \mathrm{GeV} / c)$ the identification is in both RICH detectors performed with a liquid radiator, $C_{6} F_{14}(\mathrm{n}=1.2718)$, while for higher momenta the Barrel and Forward RICH use the gas radiators $C_{5} F_{12}(\mathrm{n}=1.001941)$ and $C_{4} F_{10}(\mathrm{n}=1.001512)$ respectively. To avoid condensation of $C_{5} F_{12}$ the Barrel RICH is maintained at $40^{\circ} \mathrm{C}$.

The Cherenkov light is converted to electrons (photoelectrons) in Time Projection Drift Tubes containing the photosensitive additive TMAE (Tetrakis(diMethylAmine)Ethylene) in the drift gas.

\section{MONITORING}

The radiators $\left(C_{6} F_{14}, C_{5} F_{12}\right.$ and $\left.C_{4} F_{10}\right)$ must have a good transparency to detect the maximum 
number of photoelectrons. For this reason the fluids transmission is monitored "on line". Figure 1 presents the $C_{6} F_{14}$ transmission as a function of the wave length. The convolution of the transmission with the TMAE quantum efficiency is also shown.

A calibration system is used to measure the drift velocity of the photoelectrons in the drift gas $\left(75 \% \mathrm{CH}_{4}+25 \% \mathrm{C}_{2} \mathrm{H}_{6}\right.$ for the Barrel RICH and $\mathrm{C}_{2} \mathrm{H}_{6}$ for the Forward $\mathrm{RICH}$ ) and to observe any distortions of the electric field lines due mainly to the presence of space charge. For this purpose, spots of U.V. light are distributed into the drift tubes creating single photoelectrons at well known positions. Figure 2 shows the difference between the expected and measured spot positions on the axis perpendicular to the drift direction ( $R \Phi$ or $x-a x i s, x$ is given by the anode wire addresses) for the five spot rows placed at different drift distances. The highest distortions are expected for the last row placed at the largest drift distance $(1.5 \mathrm{~m})$ and for the spots near the drift tube border $(16.7 \mathrm{~cm}$ from the drift tube axis). In this case the difference $\Delta x$ between the expected spot position and the measured one does not exceed $1.5 \mathrm{~mm}$.

The Barrel RICH calibration system is permanently operated during the data taking periods. In order to avoid mixing of "good" photoelectrons and the ones coming from the calibration system, the U.V. lamp is pulsed only when an exclusive forward trigger $(\sim 1 \mathrm{~Hz})$ of the whole DELPHI detector is present (no tracks in the barrel region). The accuracy obtained on the drift velocity is better than $0.07 \%\left(V_{d}=5.3 \mathrm{~cm} / \mu \mathrm{s}\right.$ in the Barrel and $5.1 \mathrm{~cm} / \mu \mathrm{s}$ in the Forward RICH). The Forward RICH calibration system is also used to extract the Lorentz angle, measured to be $50^{\circ}$.

\section{PHOTOELECTRON DETECTORS}

Multi Wire Proportional Chambers (MWPC) are used to detect the photoelectrons [3]. At the operating anode-cathode voltage $\left(U_{c}=\right.$ $-1450 \mathrm{~V}$ ) a gain of $1.910^{5}$ is obtained [4]. With the discriminator thresholds used, the anode efficiency is $96 \%$ while the cathode efficiency is $86 \%$ giving an overall detection efficiency of $83 \%$.

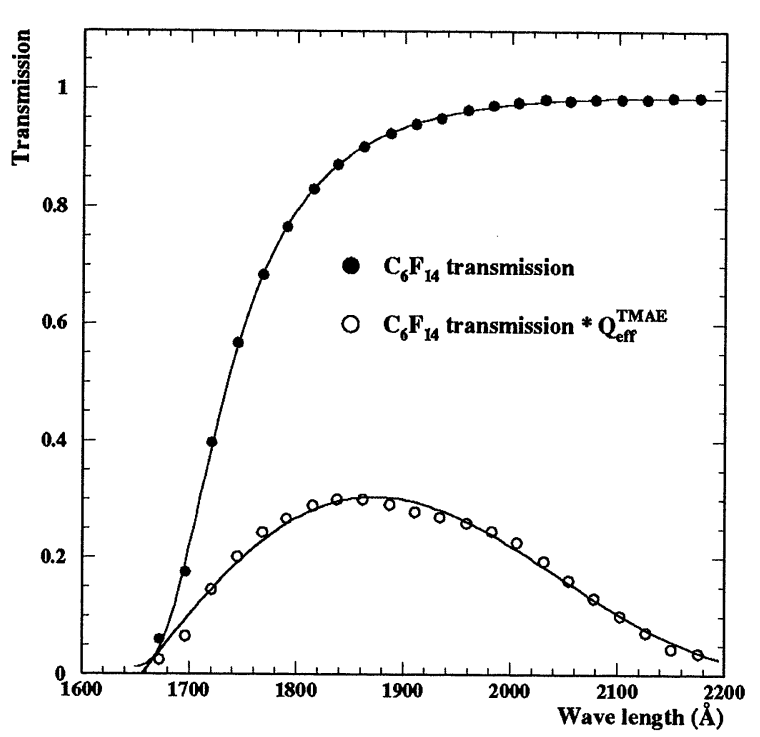

Figure 1. $C_{6} F_{14}$ transmission as a function of the wave length. The lower curve is the convolution with the TMAE quantum efficiency.

These chambers are equipped with a gating system [5] in order to be able to close the chambers after the collection of the "good" photoelectrons and prevent positive ions from drifting into the drift tubes, which could distort the electric field lines. Since no significant distortions have been observed, the gating system is just pulsed from time to time to induce signals on all anode wires and cathode strips to test all the electronic channels. The gating system could also preserve the chambers from ageing by keeping the chambers closed for non interesting events. Tests with the calibration system at the end of each running period have revealed no significant ageing effects during the 5 years of chamber operation [6].

\section{DATA ANALYSIS}

In 19941.5 million $\mathrm{Z}^{0}$ hadronic decays have been accumulated with the Barrel and Forward RICH fully operational in stable conditions. During the previous years the Barrel RICH had already collected 240000 events with both liquid and gas radiators operational and 730000 events 


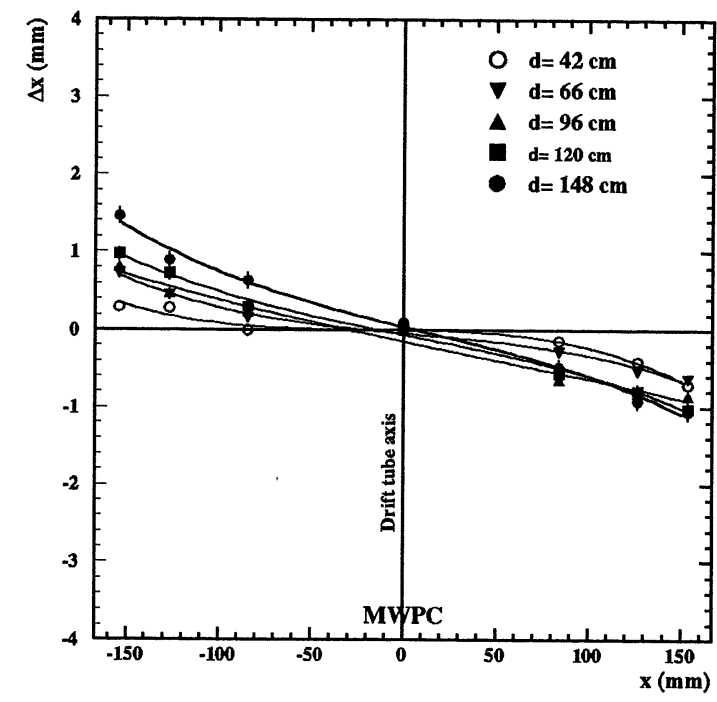

Figure 2. Difference $\Delta x$ between the expected and measured positions of the calibration system light spots in the $R \phi$ direction $(x-a x i s)$.

with only the gas radiator operational.

The candidate Cherenkov rings are first of all cleaned using software procedures $[7,8]$. Before the cleaning the ratio signal/background is of the order of $\mathbf{0 . 3 4}$ for hadronic events in the liquid radiator while after the cleaning a value of 1.5 is obtained. The signal loss due to the cleaning is limited to about $15 \%$. A powerful noise simulation program has been developed taking into account a large number of secondary effects [7] in order to reproduce as much as possible the real data. Comparing the Cherenkov angle distributions very good agreement is observed between data and simulation.

It is of prime importance to understand and reproduce by Monte Carlo simulation the Cherenkov angle resolution and the number of detected photoelectrons. Tracks from dimuon events $\left(e^{+} e^{-} \rightarrow \mu^{+} \mu^{-}\right)$saturating the Cherenkov angle $(p>40 \mathrm{GeV} / c)$ for all the radiators have been used for these studies. Figure 3 presents the Cherenkov angle resolution per photon for the Barrel RICH as a function of the mirror number (6 mirrors per side w.r.t. the interaction point placed at $84.8^{\circ}, 76.5^{\circ}, 68.7^{\circ}, 61.5^{\circ}, 54.9^{\circ}, 48.4^{\circ}$ from the beam axis) for the liquid and gas radiator compared to the expected values. For the liquid radiator a mean difference of $2 \mathrm{mrad}$ between the two values is observed. This difference is probably due to a number of small effects as for example the track direction accuracy, the RICH alignment w.r.t. the tracking detectors and the alignment of the drift tubes with each other (saturated rings from the liquid radiator cover two to three drift tubes). For the gas rings a very good agreement is obtained between real and simulated data.

1994 DELPHI BARREL RICH RESOLUTION

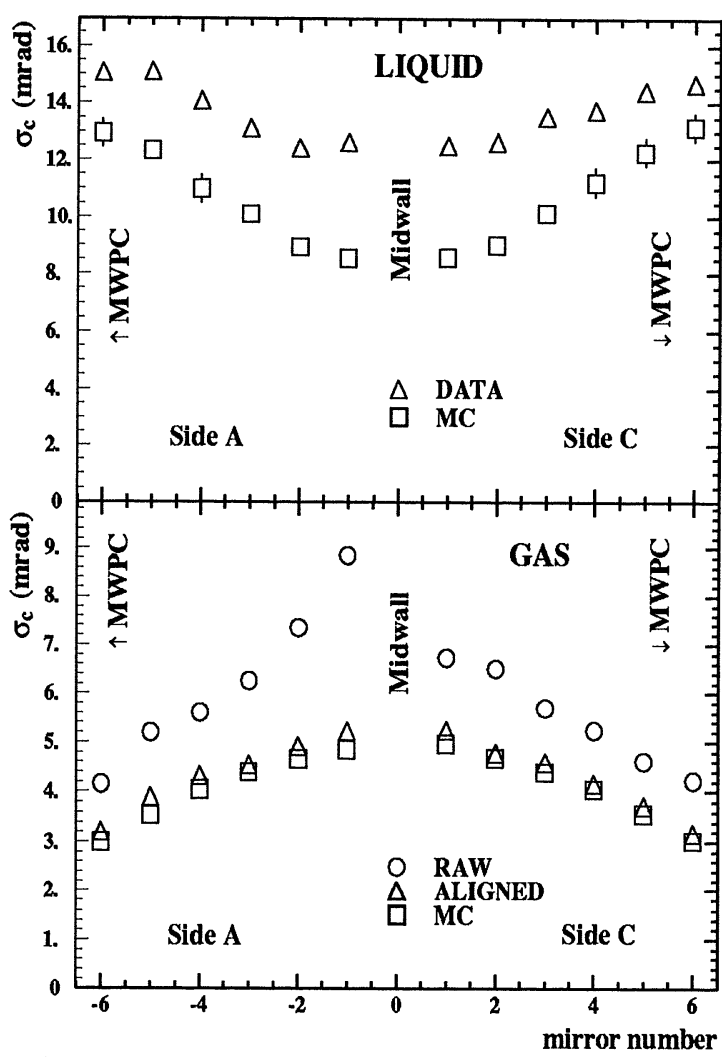

Figure 3. Cherenkov angle resolution per photon for liquid and gas radiators for saturated rings compared to the expected values for the polar angles covered by each Barrel RICH mirror. 
Figure 3 shows also for real data the Cherenkov angle resolution before and after alignment where a big improvement is observed [9]. For the Forward RICH a good agreement is also observed for the gas rings between real and simulated data concerning the Cherenkov angle resolution. For the liquid radiator the observed resolution is $11.4 \mathrm{mrad}$ while the expected value is $7.6 \mathrm{mrad}$. The difference is probably due to the same reasons as for the Barrel RICH. In order to have the same effect in both simulated and real data and be able to use the simulation to estimate the particle identification efficiencies, the simulated data are smeared in the further analysis.

The number of observed photoelectrons in the Barrel RICH as a function of the drift distance for saturated gas rings is shown by figure 4. A good agreement between real data and Monte Carlo is observed. These numbers are used to extract the electron mean free path inside the drift gas. It was found to be $4 \mathrm{~m}$ for TMAE at $28^{\circ} \mathrm{C}$ (the U.V. mean free path at this temperature is $1.8 \mathrm{~cm}$ ). The electron absorption during drift is much less crucial in the Forward than in the Barrel RICH because of the short drift distance (maximum $45 \mathrm{~cm}$ ). The mean number of photoelectrons observed per liquid ring in the Barrel RICH (without correcting for total internal reflection and dead spaces) has been found to be in a good agreement with the expected value (12.0 and 12.4 respectively). In the Forward RICH 6.9 and 7.1 photoelectrons per saturated ring from the liquid and the gas radiators respectively are observed. The expected values are 9.3 and 6.5 respectively. The difference for the liquid radiator might be due to the incomplete description in the simulation of the complicated Forward RICH geometry.

The stability in time of the number of photoelectrons, of the mean Cherenkov angle (radiator refractive index) and of its resolution are also monitored with saturated rings from leptonic events. Observed variations are taken into account by the analysis. Figure 5 presents the variation with time (in days) of the Cherenkov angle of the Forward RICH liquid radiator $\left(C_{6} F_{14}\right)$ for the whole 1994 period.

Several algorithms have been developed for the

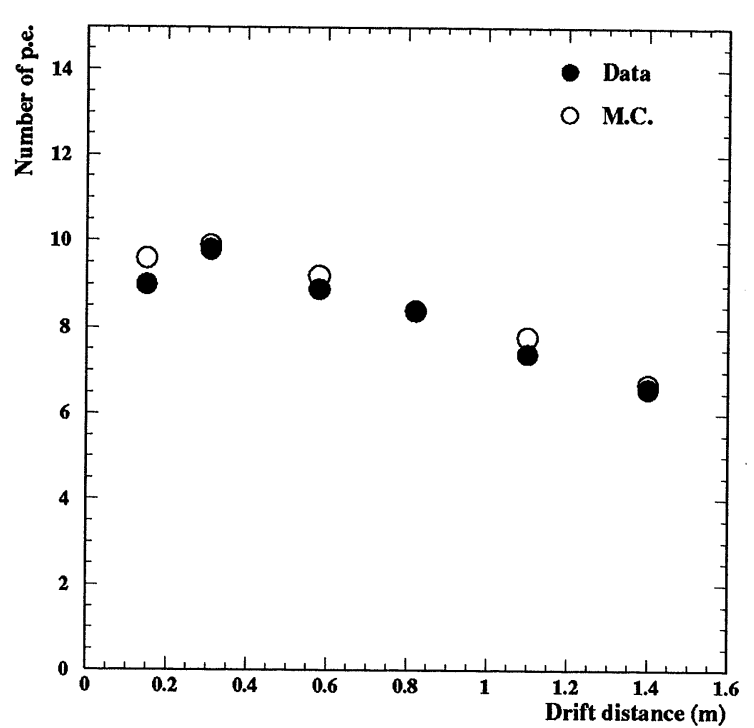

Figure 4. Number of photoelectrons for rings produced by the gas radiator as a function of the drift distance compared to the expected numbers.

particle identification [10-12]. High efficiencies and rejection factors have been obtained, e.g higher than $70 \%$ kaon identification efficiency for a pion rejection factor higher than 10. Figure 6 presents the mean Cherenkov angle for the gas radiator of the Barrel RICH versus the particle momentum. Clear pion, kaon and proton bands are observed.

\section{CONCLUSION}

Since 1994 the entire DELPHI RICH system is fully operational in stable conditions. For the gas radiators $\left(C_{5} F_{12}\right.$ and $\left.C_{4} F_{10}\right)$ the obtained performances are in agreement with the expected ones. For the liquid radiator the expected number of photoelectrons has been reached for the Barrel RICH, while in the Forward RICH the observed number is $25 \%$ lower than the expected value. The Cherenkov angle resolution from the liquid radiator for both RICH detectors is worse than the expected one due to remaining systematic effects. The particle identification provided by the DELPHI RICH is extensively used to ex- 


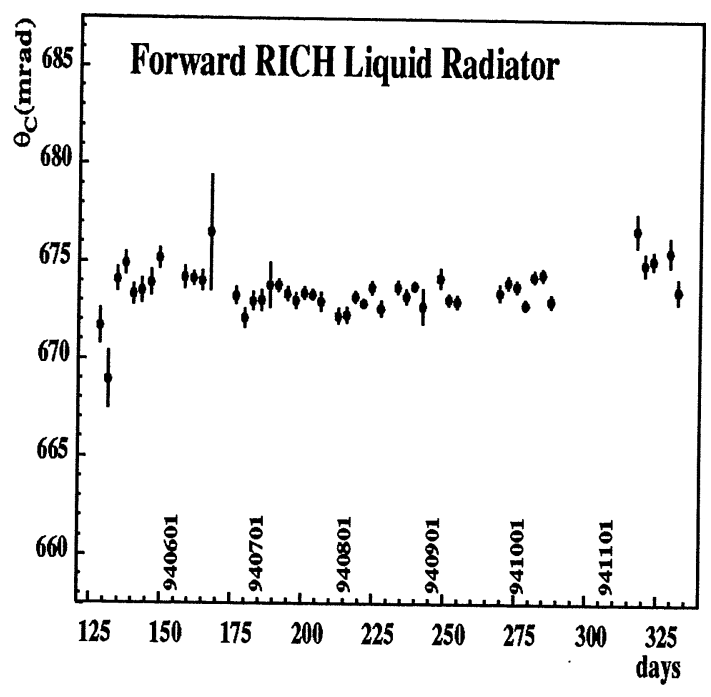

Figure 5. Variation in time of the mean Cherenkov angle for saturated rings from the Forward RICH liquid radiator.

tract physics results[13].

\section{REFERENCES}

1 E.G. Anassontzis et al, Nucl. Instr. and Meth. A323(1992)351.

2 W. Adam et al, Nucl. Instr. and Meth. A338(1994)284.

3 D. Bloch et al, Nucl. Instr. and Meth. A273(1988)847.

4 M.Dracos and D.Loukas, Nucl. Instr. and Meth. A302(1991)241.

5 G. Van Apeldoorn et al, Nucl. Instr. and Meth. A283(1989)767.

6 G. Van Apeldoorn et al., " $A$ qualitative method to determine the loss of efficiency in the DELPHI Barrel RICH MWPC's due to ageing", 3rd London Conference on Position Sensitive Detectors, 1993, Nucl. Instr. and Meth. A348(1994)249.

G. Van Apeldoorn et al., " $A$ method to measure possible ageing effects in the DELPHI Barrel RICH MWPC's; Results.", this work-

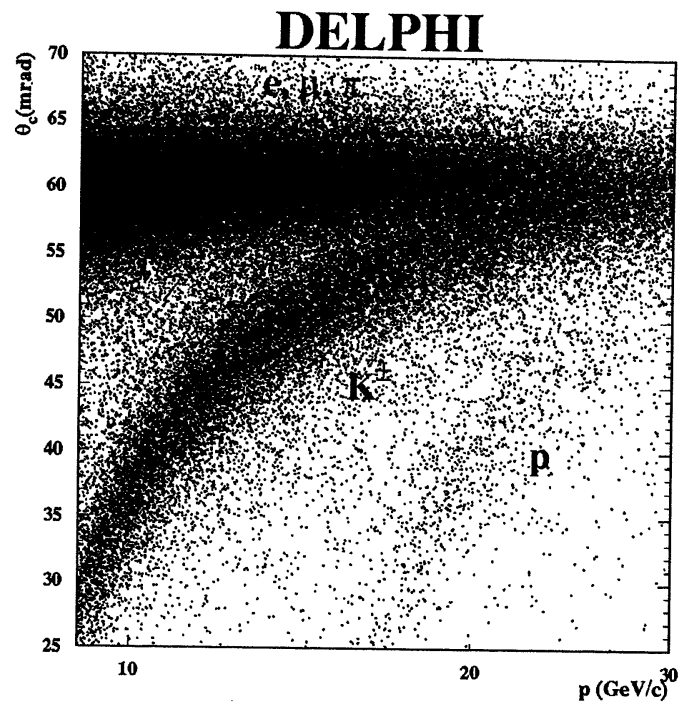

Figure 6. Mean Cherenkov angle versus the particle momentum for the Barrel RICH gas radiator.

shop, presented by D. Toet.

7 D. Bloch et al., "Noise simulation and rejection for the DELPHI Ring Imaging Cherenkov detector", this workshop, presented by M. Dracos.

8 J. Garcia Perez et al., "Results from Background Reduction in the DELPHI Forward $R I C H "$, this workshop, presented by J. Garcia Perez.

9 W. Adam et al, "Alignment and Cherenkov angle resolution of the DELPHI RICH", this workshop, presented by J. Medbo.

10 P. Baillon, Nucl. Instr. and Meth. A238(1985)341.

11 M. Dracos, "A particle identification method for Ring Imaging Cherenkov detectors", CRN 95-21.

12 W. Adam et al, "Particle identification algorithms for the DELPHI RICH detector", this workshop, presented by W. Adam.

13 See contributions on physics results from DELPHI collaboration presented at this workshop. 